\title{
TITLE:
}

\section{Characterisation of circadian rhythms of various duckweeds.}

$\operatorname{AUTHOR}(S)$ :

Muranaka, T; Okada, M; Yomo, J; Kubota, S; Oyama, $\mathrm{T}$

\section{CITATION:}

Muranaka, T ... [et al]. Characterisation of circadian rhythms of various duckweeds.. Plant biology 2015, 17(Supplement s1): 66-74

ISSUE DATE:

2015-01

URL:

http://hdl.handle.net/2433/199678

\section{RIGHT:}

This is the peer reviewed version of the following article: Muranaka, T., Okada, M., Yomo, J., Kubota, S., Oyama, T. (2015), Characterisation of circadian rhythms of various duckweeds. Plant Biology, 17:66-74, which has been published in final form at http://dx.doi.org/10.1111/plb.12202. This article may be used for non-commercial purposes in accordance with Wiley Terms and Conditions for Self-Archiving; This is not the published version. Please cite only the published version.; この論文は出版社版でありません。引用の際には出版社版をご確認ご利用ください。 
Title: Characterization of circadian rhythms of various duckweeds

Running head: Circadian rhythms of duckweeds

\section{Authors}

Tomoaki Muranaka, Masaaki Okada, Jun Yomo, Saya Kubota, Tokitaka Oyama

Department of Botany, Graduate School of Science, Kyoto University, Kitashirakawa-oiwake-cho, Sakyo-ku, Kyoto 606-8502, Japan

\section{Corresponding author:}

Name: Dr. Tokitaka Oyama

Address: Department of Botany, Graduate School of Science, Kyoto University, Kitashirakawa-oiwake-cho, Sakyo-ku, Kyoto 606-8502, Japan

Phone: +81 757534135

Fax: $\quad+81757534137$

E-mail:oyama@cosmos.bot.kyoto-u.ac.jp

\section{Key words:}

Duckweeds, Circadian rhythm, Diversity, Luciferase reporter, Bioluminescence monitoring, CCA1, UBIQUITIN 


\section{Abbreviations}

CCA1, CIRCADIAN CLOCK ASSOCIATED1; LHY, LATE ELONGATED HYPOCOTYL; PRR, PSEUDO RESPONSE REGULATOR; TOC1, TIMING OF CAB EXPRESSION1; GI, GIGANTIA; ELF3, EARLY FLOWERING 3; UBQ1, UBIQUITIN1; CaMV35S, cauliflower mosaic virus 35S; FFT-NLLS, fast Fourier transform-nonlinear least squares; RAE, relative amplitude error; SEM, standard error of the mean 


\section{ABSTRACT}

The plant circadian clock controls various physiological phenomena which are important for adaptation to natural day-night cycles. Many components of the circadian clock have been identified in Arabidopsis thaliana (Arabidopsis), the model plant for molecular genetic studies. Recent studies revealed evolutionary conservation of clock components in green plants. Homologues of clock related genes have been isolated from Lemna gibba and Lemna aequinoctialis, and it has been demonstrated that these homologues function in the clock system in a manner similar to their functioning in Arabidopsis. While clock components are widely conserved, circadian phenomena display diversity even within the Lemna genus. In order to survey the full extent of diversity in circadian rhythms among duckweed plants, we characterized the circadian rhythms of duckweed plants by employing a semi-transient bioluminescent reporter system. Using a particle bombardment method, circadian bioluminescent reporters were introduced into nine strains representing five duckweed species: Spirodela polyrhiza, Landoltia punctata, Lemna gibba, Lemna aequinoctialis, Wolffia columbiana. We then monitored luciferase (luc+) reporter activities driven by AtCCA1-, ZmUBQ1-, CaMV35S promoters under entrainment- and free-running conditions. Under entrainment conditions, AtCCA1::luc+ showed similar diurnal rhythms in all strains. This suggests that the mechanism of biological timing under day-night cycles is conserved throughout the evolution of duckweed plants. Under free-running conditions, we observed circadian rhythms of AtCCA1::luc+, ZmUBQ1::luc+ and CaMV35S::luc+. These circadian rhythms showed diversity in period length and sustainability, suggesting that circadian clock mechanisms are somewhat diversified among duckweed plants. 


\section{INTORODUCTION}

The circadian clock, a self-sustaining oscillator with a period of approximately $24 \mathrm{~h}$, is ubiquitous across life, including cyanobacteria, plants, insects, and mammals. It is synchronized to day-night cycles and controls various biological phenomena to adapt organisms to diurnal environmental changes (Dunlap et al. 2005). The plant circadian clock controls various physiological behaviors such as leaf movements, stomatal movements, metabolism, and photoperiodic flowering (Barak et al. 2000). The circadian clockwork is important for optimizing productivity, starch utilization, seed yield and seed viability (Green et al. 2002; Dodd et al. 2005; Graf et al. 2010). Understanding the mechanism of circadian regulation in plants is therefore important for improving plant performance in agriculture, as well as for applications such as bioremediation.

In plants, the circadian clock system consists of interconnected transcription-translation feedback loops (Pokhilko et al. 2013). Components of these feedback loops have been identified in Arabidopsis, the model plant for molecular genetic studies (Nakamichi 2011). Recent studies revealed evolutionary conservation of clock components within plants. In particular, homologues to myb domain transcription factors CIRCADIAN CLOCK ASSOCIATED1 (CCA1), LATE ELONGATED HYPOCOTYL (LHY), and the PSEUDO RESPONSE REGULATOR (PRR) family, including TIMING OF CAB EXPRESSION1 (TOC1) were identified in species as diverse as green algae (Ostreococcus tauri), moss (Physcomitrella patens), and many higher plants (McClung 2013). Characterization of these homologues in many species provided additional evidence of conserved clock architecture (Song et al. 2010). Circadian clock systems of duckweed plants have been well analyzed for a long time. Physiological rhythms, such as $\mathrm{CO}_{2}$ output and potassium uptake, were monitored in duckweed plants, and their circadian traits were characterized (Miyata and Yamamoto 1969; Hillman 1970; Kondo and Tsudzuki 1978). As clock-related genes, LHY, PRRs, GI and ELF3 have been identified in Lemna gibba and Lemna aequinoctialis (Lemna paucicostata), and a semi-transient bioluminescent reporter system introduced by particle bombardment has been successfully applied to analyze Lemna circadian systems (Miwa et al. 2006). Functional assessment using a reporter-effector assay (knockdown and overexpression) of these homologues revealed functional conservation of clock components between L. gibba and Arabidopsis (Serikawa et al. 2008). While clock components are widely conserved in plants, circadian phenomena show diversity even within the Lemna genus (Kondo and Tsudzuki 1978; Miwa et al. 2006). Under constant light 
conditions, L. gibba demonstrate clear circadian rhythms but $L$. aequinoctialis does not.

In this study we aimed to capture the full extent of diversity in circadian rhythms among duckweed plants. Duckweed plants are suitable for comparative analysis of circadian systems because, as described above, the clock systems of Lemna plants have been well characterized and duckweed circadian rhythms are easily monitored by employing a semi-transient bioluminescent reporter system (Miwa et al. 2006; Serikawa et al. 2008). When the semi-transient bioluminescent reporter system is applied for monitoring circadian rhythms of various plants, short handling time for gene transfection by particle bombardment and its versatility are remarkable advantages. Furthermore, tiny and flat duckweed plants grow well in a small dish for a long period. These advantages allow us to employ an automated bioluminescent monitoring system for high throughput characterization of circadian rhythms in duckweed plants. Many duckweed strains of various species are capable of being cultured as intact mature plants in the same, strictly controlled conditions in the laboratory (Landolt 1986). This feature is necessary for an accurate comparison of circadian clock systems since circadian rhythms in higher plants are affected by culture conditions (Chen et al. 2013; Kusakina et al. 2014). Thus, using a particle bombardment method for the circadian bioluminescent reporter system, duckweed plants provide a convenient platform for an analysis of natural variations in circadian clock systems.

We characterized circadian rhythms in nine strains representing five duckweed species and four genera; Lemna gibba G3 and p8L, Lemna aequinoctialis 6746 and Nd, Landoltia punctata NS and IS, Spirodela polyrhiza 7003 and biwa1, Wolffia columbiana 7972 (Fig. S1 and S2). The circadian rhythms of L. gibba G3 and L. aequinoctialis 6746 were previously analyzed (Miyata and Yamamoto 1969, Hillman 1970, Kondo and Tsudzuki 1978; Miwa et al. 2006; Serikawa et al. 2008; Muranaka et al. 2013). Bioluminescent reporter genes were introduced into the nine strains by a particle bombardment method and reporter activities were monitored under entrainment and free-running conditions. Under entrainment conditions, reporter activities showed similar diurnal rhythms in all strains. Under free-running conditions, circadian rhythms showed diversity in period length and sustainability, suggesting that circadian clock systems are somewhat diversified among duckweed plants.

\section{MATERIALS AND METHODS}




\section{Plant materials and growth conditions}

Lemna gibba G3 strain and Lemna aequinoctialis 6746 strain (previously called Lemna paucicostata 6746 or Lemna perpusilla 6746) were used as previously described (Miwa et al. 2006). L. gibba p8L strain is a pure line (eight generations of selfing) produced from the G3 strain. Spirodela polyrhiza 7003 and Wolffia columbiana 7972 strains originated from the Landolt collections and were the kind gifts of Dr. Todd Michael. L. aequinoctialis Nd (original strain), Landoltia punctata NS and IS and S. polyrhiza biwa1 strains were isolated in our laboratory. The S. polyrhiza biwa1 strain was collected at Shiga, Japan and the others were collected at Kyoto, Japan. L. aequinoctialis Nd strain is a pure line (six generations of selfing) of the original strain. L. gibba plants were kept in E medium with $1 \%$ sucrose; other plants were kept in $0.5 \times \mathrm{H}$ medium with $1 \%$ sucrose. Plants were maintained in the same growth medium as that used in the bioluminescence monitoring experiments for at least three weeks before starting the monitoring. E medium was made by adding $30 \mu \mathrm{M}$ ethylene-di-amine-tetra-acetic acid (EDTA) to M medium (Cleland and Briggs 1968). The compositions of M and H medium were described by Hillman (1961). The composition of NF

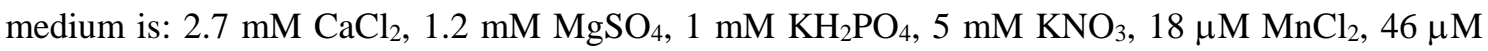
$\mathrm{H}_{3} \mathrm{BO}_{3}, 0.77 \mu \mathrm{M} \mathrm{ZnSO}_{4}, 0.32 \mu \mathrm{M} \mathrm{CuSO}_{4}, 0.49 \mu \mathrm{M} \mathrm{MoO}_{3}, 20 \mu \mathrm{M} \mathrm{FeSO}_{4}, 50 \mu \mathrm{M} \mathrm{Na}_{2}$ EDTA. The pH was adjusted to 5.0 with $\mathrm{KOH}$. All strains were cultured under constant light conditions; light intensity supplied by fluorescent lamps (FLR40SEX-W/M/36-HG; NEC, http://www.nec.com/) was approximately $50 \mu \mathrm{E} \mathrm{m}^{-2} \mathrm{~s}^{-1}$. Growth temperature was maintained at $25^{\circ} \mathrm{C} \pm 1^{\circ} \mathrm{C}$. Colonies were grown in $75 \mathrm{ml}$ of growth medium in $200 \mathrm{ml}$ Erlenmeyer flasks plugged with cotton. New stock cultures were made every week, and well-grown plants were used for experiments.

\section{Species identification}

The species of the strains isolated in our laboratory were identified by morphological characteristics (Les et al. 1997). To confirm species identification, the atpF-atpH marker was sequenced (Wang et al. 2010). Total DNA of each strain was extracted using Extract-N-Amp Plant PCR Kits (SIGMA-ALDRICH, http://www.sigmaaldrich.com/). The atpF-atpH sequences were amplified with primers as described in Wang et al. (2010). PCR products were purified with GenElute PCR Clean-Up Kit (SIGMA-ALDRICH, http://www.sigmaaldrich.com/) and then sequenced with an Applied Biosystems 3130xl Genetic Analyzer (Applied Bisosystems, http://www.appliedbiosystems.com/). The sequence of each strain was compared with the reference 
sequences reported in Wang et al. (2010) and a phylogenetic tree was generated using ClustalW (http://clustalw.ddbj.nig.ac.jp/) (Fig. S1).

\section{Luciferase reporter constructs}

pUC-AtCCA1::luc+ (AtCCA1::luc+) was used as the circadian bioluminescent reporter (Muranaka et al. 2013). pSP1-ZmUBQ1::luc+ (ZmUBQ1::luc+) and pSP1-AtPRR1::luc+ (AtPRR1::luc+) were described previously (Miwa et al. 2006; Serikawa et al. 2008). pUC18-CaMV35S::intron-luc+ (CaMV35S::luc+) is a modified version of pUC18-CaMV35S::luc+, which has been described previously (Muranaka et al. 2013), with an intron sequence inserted at 148 bp from the start codon of the luciferase gene. The inserted intron sequence was described in Ohta et al. (1990).

\section{Particle bombardment experiment}

Reporter constructs were introduced into plants by particle bombardment. $8 \mu \mathrm{l}$ of prewashed gold particle suspension (1 $\mu \mathrm{m}$ diameter; Bio-Rad, http://www.bio-rad.com/) in 50\% glycerol (60 mg $\mathrm{ml}^{-1}$ ) was mixed with $2 \mu \mathrm{l}$ of plasmid DNA $\left(1 \mu \mathrm{g} \mu \mathrm{l}^{-1}\right)$, $3.3 \mu \mathrm{l}$ of spermidine $(0.1 \mathrm{M})$, and $8 \mu \mathrm{l}$ of $\mathrm{CaCl}_{2}(2.5 \mathrm{M})$. After incubating for 5 minutes at room temperature, the suspension was centrifuged for $10 \mathrm{~min}$ at 12,000 rpm at room temperature and the supernatant was discarded. DNA-coated particles were washed once with $80 \mu \mathrm{l}$ of $80 \%$ ethanol and once with $100 \%$ ethanol and resuspended in $15 \mu \mathrm{l}$ of $100 \%$ ethanol. The suspension of DNA-coated particles were then spread on a macrocarrier and dried. A helium gun device (PDS-1000/He; Bio-Rad) was used according to the manufacturer's instructions [vacuum, 28 mmHg; helium pressure, 450 psi (rupture disc)]. Duckweed plants were laid on a 60-mm polystyrene dish (IWAKI, http://www.iwaki-kk.co.jp/). The dish was set underneath the muzzle of the gun, and DNA-coated particles on the macrocarrier were fired into the plants. After bombardment, duckweed plants were divided into four $35-\mathrm{mm}$ dishes with $3 \mathrm{ml}$ of growth medium containing firefly luciferin $(0.5 \mathrm{mM}$ potassium salt; Wako, http://www.wako-chem.co.jp/).

\section{Bioluminescence monitoring}

Bioluminescence monitoring was performed as described previously (Miwa et al. 2006; Serikawa et al. 2008) with minor revisions. The luminescence dish-monitoring system used photomultiplier tubes (H7360-01; Hamamatsu Photonics K.K.) for bioluminescence detection. To reduce background 
chlorophyll fluorescence, a short-pass filter (SVO630; Asahi Spectra) was set at the detection site of the photomultiplier tubes. Each dish was subjected to $30 \mathrm{~s}$ measurement every $20 \mathrm{~min}$. The monitoring system was placed in an incubator (KCLP-1000I-CT; NK system, http://www.nihonika.co.jp/) with fluorescent lamps (FL20SSW/18; Mitsubishi Electric co., http://www.mitsubishielectric.com/). Temperature was maintained at $25^{\circ} \mathrm{C} \pm 1{ }^{\circ} \mathrm{C}$ and light intensity was approximately $30 \mu \mathrm{E} \mathrm{m}^{-2} \mathrm{~s}^{-1}$.

\section{FFT-NLLS analysis}

In order to correctly estimate period lengths and relative amplitude errors, the recorded bioluminescence under constant conditions (48-168 h) were detrended by applying moving average filter (24-h window size) and then analyzed with the FFT-NLLS suite of programs available within the Biological Rhythms Analysis software system (BRASS, available from http://www. amillar.org).

\section{RESULTS}

\section{A semi-transient bioluminescent reporter system for monitoring circadian rhythms in duckweed plants.}

A bioluminescent reporter system using firefly luciferase has been successfully used to characterize circadian rhythms in plant, animal and cyanobacterial cells (Welsh et al. 2005; Ishiura et al. 1998). We employed a semi-transient bioluminescent reporter system to analyze the circadian rhythms of duckweed plants (Miwa et al. 2006; Serikawa et al. 2008). Reporter constructs were introduced into plants by particle bombardment. Transfected plants were placed in an automated bioluminescence monitoring system in which bioluminescence was measured by a sensor equipped with a photomultiplier tube every 20 minutes. During monitoring, plants were first entrained to two $12 \mathrm{~h}$ dark / 12 h light cycles, after which they were released into constant light or constant dark conditions. Circadian period lengths under constant conditions were estimated by fast Fourier transform-nonlinear least squares (FFT-NLLS) analysis. In FFT-NLLS analysis, the degree of confidence is described by the relative amplitude error (RAE), which ranges from 0 (complete sine wave) to 1 (arrhythmic) (Plautz et al. 1997).

Luciferase driven by the Arabidopsis CCA1 promoter (AtCCA1::luc+) was used to measure circadian rhythms in Lemna plants (Miwa et al. 2006; Serikawa et al, 2008; Muranaka et al. 
2013). We introduced AtCCA1::luc+ into L. gibba G3 and S. polyrhiza 7003 strains. In L. gibba plants, AtCCA1::luc + reporter activities showed diurnal rhythms under $12 \mathrm{~h}$ dark / $12 \mathrm{~h}$ light cycles and circadian rhythms under constant light conditions in both $\mathrm{E}$ and $0.5 \mathrm{x} \mathrm{H}$ growth media (Fig. 1A and B). However, bioluminescence traits were different in $\mathrm{E}$ medium and $0.5 \times \mathrm{H}$ medium. In $\mathrm{E}$ medium, the estimated period length under constant light conditions was $22.62 \pm 0.07$ (mean \pm SEM, $\mathrm{n}=4$ ) and the bioluminescence level gradually decreased (Fig 1A). In $0.5 \times \mathrm{H}$ medium, the estimated period length was $24.26 \pm 1.01(\mathrm{n}=4)$ and the bioluminescence level was maintained during monitoring (Fig 1B). Bioluminescence of AtCCA1::luc+ in S. polyrhiza plants was low when cultured in $0.5 \times \mathrm{H}$ medium (Fig. 1C). These data suggest that bioluminescence rhythms in duckweed plants are influenced by growth medium conditions. We modified the composition of the growth medium to create NF medium, in which the bioluminescence level of AtCCA1::luc+ in every tested duckweed plant was high enough to characterize temporal changes clearly. Every tested duckweed plant was well-grown in NF medium (Fig. S2).

\section{Diurnal rhythms of five duckweed species across four genera}

In addition to the circadian reporter, AtCCA1::luc+, luciferase driven by the maize UBIQUITIN1 promoter (ZmUBQ1::luc+) was used as a constitutive reporter in Lemna plants (Miwa et al. 2006; Serikawa et al, 2008). We introduced AtCCA1::luc+ or ZmUBQ1::luc+ into nine strains representing five duckweed species across four genera; L. gibba G3 and p8L, L. aequinoctialis 6746 and Nd, L. punctata NS and IS, S. polyrhiza 7003 and biwa1, W. columbiana 7972 (Fig S1). AtCCA1::luc + showed a clear diurnal rhythm in each strain under the $12 \mathrm{~h}$ dark / $12 \mathrm{~h}$ light cycle (Fig. 2A-I). Reporter activity peaked in the morning phase, as observed in Arabidopsis and in Lemna plants cultured in different medium (Nakamichi et al. 2004; Miwa et al. 2006). Peak times were different between Lemna species (0-2 h after dawn; Fig. 2A-D) and other genera (3-4 h after dawn; Fig2E-I) (Table 1). ZmUBQ1::luc+ reporter activity did not show a clear diurnal rhythm in any duckweed plant (Fig. 2J-R). Peak bioluminescence intensities of either reporter were similar between strains of the same species, while they were different between strains of different species (Table 1). Note that bioluminescence in L. aequinoctialis $\mathrm{Nd}$ showed an acute increase at the dark-light transition (Fig. 2D and M). L. aequinoctialis Nd and W. columbiana 7972 strains showed high bioluminescence levels for both reporters. 


\section{Circadian rhythms of five duckweed species across four genera under constant light conditions}

Reporter activities of AtCCA1::luc+ and ZmUBQ1::luc+ were monitored in the nine strains under constant light conditions. AtCCA1::luc+ showed circadian rhythmicity with similar period lengths in L. gibba G3 and p8L (Fig. 3A and B, Table 2). ZmUBQ1::luc+ activity showed damped circadian rhythms with four peaks (Fig. $3 \mathrm{~J}$ and K). These peak times corresponded to the phase of subjective dawn. The estimated period lengths of ZmUBQ1::luc+ rhythms were $\sim 1 \mathrm{~h}$ longer than those of AtCCA1::luc+ (Table 2). The circadian rhythmicity of ZmUBQ1::luc+ was unexpected, so we tested CaMV35S::luc+, wherein luciferase expression is driven by the commonly used constitutive cauliflower mosaic virus 35S promoter (Leeuwen et al. 2000). Under constant light conditions, CaMV35S::luc+ showed a damped circadian rhythm with three peaks, at which ZmUBQ1::luc+ reporter activities peaked in L. gibba p8L (Fig. S3). The estimated period length was $25.59 \pm 0.08 \mathrm{~h}$ $(\mathrm{n}=4)$, similar to that of the ZmUBQ1::luc+ $(25.63 \mathrm{~h})$ in the same strain (Table 2). Thus, these reporters which typically show constitutive expression seemed to function as novel circadian reporters in our experimental conditions. It is noteworthy that AtPRR1::luc+ reporter activities in $L$. gibba under the same conditions showed a circadian rhythm in antiphase to those of AtCCA1::luc+, ZmUBQ1::luc+, and CaMV35S::luc+ (Fig. S4; Serikawa et al. 2008). This suggests that the circadian rhythmicity of them was unlikely to result from a possible genome-wide transcriptional regulation with circadian rhythmicity.

Characteristics of AtCCA1::luc+ and ZmUBQ1::luc+ bioluminescence rhythms were different between L. aequinoctialis 6746 and L. aequinoctialis Nd strains. In the 6746 strain, AtCCA1::luc+ reporter activity showed a severely damped circadian rhythm with only one peak, while in the Nd strain, reporter activity showed a gradually damped rhythm with five peaks. (Fig. 3C and D). ZmUBQ1::luc+ showed a gradual decrease without clear circadian rhythmicity in the 6746 strain $(\mathrm{RAE}=0.27)$ and a low-amplitude circadian rhythm with four peaks in the Nd strain $(\mathrm{RAE}=$ 0.20) (Fig. 3L and M, Table 2). The estimated period lengths of the AtCCA1::luc+ rhythm (21.55 h) and the ZmUBQ1::luc+ rhythm (21.76 h) in the Nd strain were similar (Table 2). In both strains, bioluminescence intensity from AtCCA1::luc+ transfected cells was relatively low under constant light conditions when compared to the intensity under the $12 \mathrm{~h}$ dark / $12 \mathrm{~h}$ light cycle.

AtCCA1::luc+ showed circadian rhythms in Landoltia punctata NS and IS strains (Fig. $3 \mathrm{E}$ and F). The estimated period length of the NS strain (22.39 h) was $\sim 0.5 \mathrm{~h}$ shorter than that of the 
IS strain (22.91 h) (Table 2). ZmUBQ1::luc+ reporter activity also showed a damped circadian rhythm with five peaks in L. punctata NS and IS strains (Fig. 3N and O). Its period length was similar to that of AtCCA1::luc + in both strains (Table 2).

AtCCA1::luc+ showed damped circadian rhythms with three peaks in S. polyrhiza 7003 and biwa1 strains (Fig. 3G and H). The estimated period length of the 7003 strain (25.84 h) was 1.5 $\mathrm{h}$ longer than that of the biwa1 strain (24.42 h) (Table 2). ZmUBQ1::luc+ showed damped circadian rhythms with three peaks in S. polyrhiza 7003 and biwa1 strains (Fig. 3P and Q) with a period length $\sim 0.5 \mathrm{~h}$ longer than that of the AtCCA1::luc + rhythm in the 7003 strain, while period lengths of both bioluminescent reporter rhythms were similar in the biwa1 strain (Table 2).

AtCCA1::luc+ showed damped circadian rhythm with four peaks in the W. columbiana 7972 strain (Fig. 3I). The first peak interval ( $\sim 30 \mathrm{~h})$ was larger than the second and third intervals ( $20 \mathrm{~h}$ ), indicating that the period length of the circadian rhythm was unstable. ZmUBQ1::luc+ did not show a clear circadian rhythm in this strain (Fig. 3R).

\section{Circadian rhythms of five duckweed species across four genera under constant dark conditions}

AtCCA1::luc+ and ZmUBQ1::luc+ activities were monitored in the nine strains under constant dark conditions. AtCCA1::luc+ showed a damped circadian rhythm in each strain (Fig. 4A-I). The estimated period length was longer than that under constant light conditions in each strain except $W$. columbiana 7972, in which the estimated period length was shorter (Tables 2 and 3). However, as mentioned above, the AtCCA1::luc+ period length in W. columbiana 7972 strain was unstable under constant light conditions, and peak intervals of the second and third cycles ( 20 h and $\sim 22 \mathrm{~h}$, respectively) were shorter than the estimated period length under constant dark conditions ( $\sim 24 \mathrm{~h}$ ). Thus the circadian period lengths of duckweed plants were likely to be longer under constant dark conditions than under constant light conditions, as observed in Arabidopsis (Dalchau et al. 2011). ZmUBQ1::luc+ activity gradually decreased without clear rhythmicity (RAE $>0.25)$ in each strain (Fig. 4J-R, Table 3). Since ZmUBQ1::luc+ showed circadian rhythms under constant light conditions, the bioluminescence rhythm of ZmUBQ1::luc+ appears to be light dependent.

\section{DISCUSSION}

In this study we investigated the diversity of circadian systems in duckweed plants by monitoring 
circadian rhythms in nine duckweed strains representing five species (Lemna gibba, Lemna aequinoctialis, Landoltia punctata, Spirodela polyrhiza, Wollfia columbiana) with a semi-transient bioluminescent reporter system. Under constant conditions, circadian AtCCA1::luc+ rhythms showed diversity in period lengths and sustainability. Period lengths were varied under both constant light (21.55-25.84 h) and constant dark conditions (23.59-29.71 h) (Tables 2 and 3). The number of observable peaks ranged from one to five (Fig. 3 and 4). This suggests that the circadian clock mechanisms are somewhat diversified among duckweed plants. The range of period lengths between strains of the same species $(<2 \mathrm{~h})$ was smaller than that between strains of different species and was consistent with natural variation in Arabidopsis (Tables 2 and 3) (Micheal et al, 2003). In contrast to period length, sustainability showed diversity even between strains of the same species. The number of observable peaks of AtCCA1::luc+ activity in L. aequinoctialis was different between the 6746 strain (one peak) and the Nd strain (five peaks) under constant light conditions (Fig. 3C and D). It should be noted that both strains showed three peaks under constant dark conditions (Fig. 4 C and D). The light dependency of these differences in sustainability implies that this phenotypic diversity might be caused by differences in light signaling mechanisms between the two strains. In $L$. aequinoctialis (L. paucicostata) species, a short-day photoperiodic flowering species, the critical dark periods showed a large range of natural variation (0-13.5 h) (Yukawa and Takimoto 1976). Thus, L. aequinoctialis may show a wide range of natural variation in a time dependent manner.

Under entrainment conditions with $12 \mathrm{~h}$ dark / 12 h light cycles, AtCCA1::luc+ showed diurnal rhythms with peaks in the morning in all nine strains. This suggests that the mechanisms for scheduling biological timing in the presence of the day-night cycle are conserved in duckweed plants. However, duckweeds in the Lemna genus showed peak times $\sim 2 \mathrm{~h}$ earlier than those of the other three genera. Among these four genera, only Lemna species lack guard cell plastids, and it was shown that stomata of L. gibba plants are permanently open and do not respond to light-dark transitions (Hes et al. 1997; Park et al. 1990). One possible explanation of these peak time differences is that the lack of diurnal stomatal movements affects daily metabolic changes and the phase of morning gene expression. It should be noted that at least one peak was observed in constant conditions after the light-dark (dark-light) transition in each strain (Fig. 3A-I and 4A-I). This means that the circadian system in each strain was capable of keeping time information for at least one day after entrainment cues were removed. One of the major functions of the circadian clock system is anticipation of dawn after reset at the previous dawn (Dodd et al. 2014). Our study 
indicates that this function was conserved in the evolution of duckweed plants.

Under constant light conditions, ZmUBQ1::luc+ and CaMV35S::luc+ showed circadian rhythms with the peak time in the morning in many duckweed species (Fig. 3J-R). In Arabidopsis, the promoter activity of a UBIQUITIN gene is remarkably stable and often used as a reference gene for transcript normalization (Czechowski et al. 2005). The CaMV35S promoter is also well-known as a constitutive promoter, and the bioluminescence of CaMV35S::luc + did not show a clear circadian rhythm under constant conditions in Arabidopsis (Millar et al. 1992). Hence, the circadian rhythms of these reporters observed in duckweed plants might be generated at the level of post-transcriptional regulations (Kojima et al. 2011; Pal et al. 2013). In addition to luciferase enzyme amounts, intracellular concentrations of its substrates affect bioluminescence intensity in vivo. Firefly luciferase catalyzes the ATP-dependent oxidation of luciferin in a reaction that produces light (Leeuwen et al. 2000). In many plant species, circadian regulation of photosynthesis has been analyzed (Dodd et al. 2013). In Landoltia punctata (Spirodela oligorrhiza), it was reported that phosphorylation of the D1 photosystem II reaction center protein is controlled by an endogenous circadian rhythm (Booij-James et al. 2002). Thus, luciferase reporter activities may be influenced by changes in intracellular concentrations of ATP and oxygen, which are dependent on photosynthetic activities and may cause circadian rhythmicity of these reporters. Even though these possible rhythms would widely influence the luciferase reporter activities, bioluminescence rhythms of the AtCCA1::luc + reporter with similar peak time were likely to reflect its transcription behavior. It was reported that overexpression/RNAi of clock-related genes in the reporter-introduced cells of L. gibba changed bioluminescence traces of AtCCA1::luc+ but it did not affect those of ZmUBQ1::luc+ (Serikawa et al. 2008). Moreover, the possible rhythmic influence on the luciferase reporter would be not as large as to overcome the circadian rhythms of AtPRR1::luc+ with peak time in the evening (Fig. S4). The physiological and molecular basis for the circadian rhythms of ZmUBQ1::luc+ and CaMV35S::luc+, and the extent of this kind of phenomena across plants should be an interesting issue to understand the circadian system in plants.

In previous studies of natural variation in plant circadian systems, circadian outputs such as leaf movements and delayed fluorescence were used as markers for circadian rhythms (Micheal et al. 2003; Gould et al. 2009). A recent study using circadian luciferase reporters in various Arabidopsis accessions revealed difference in the temperature-buffering capacity between the leaf movement rhythm and the clock gene expression (Kusakina et al. 2014). In this study we monitored 
the rhythm of clock gene expression, which directly reflects features of the circadian clock, by employing a semi-transient bioluminescent reporter system using particle bombardment. In the particle bombardment method, reporter constructs are introduced into only mature epidermal( $20 \%$ ) or mesophyll ( 80\%) cells (Muranaka et al. 2013). This allowed us to characterize the circadian oscillator in plant cells without complexity due to cell proliferation and developmental processes (Fukuda et al. 2007; Yakir et al. 2009; Fukuda et al. 2012). The flat, smooth surface of the duckweed frond is suitable for the particle bombardment method and the tiny body allowed us to keep the whole plant in small dishes under normal growth conditions throughout the experiments. These characteristics of duckweed plants and the reporter transfection technique give advantages to compare circadian rhythms of many plants in various species and strains. Thus, duckweed plants are a good model for research on the divergence and evolution of circadian clock systems in higher plants.

\section{ACKNOWLEDGEMENTS}

This research was supported in part by JSPS KAKENHI Grant numbers 23657033 (T. O.), 25650098 (T. O.), 24-1530 (T. M.), by The Iwatani Naoji Foundation (T. O.), by ALCA JST (T. O.) and by PRESTO JST (T. O.). We thank Drs. Kumiko Miwa (Nagoya Univ., Nagoya), Norihito Nakamichi (Nagoya Univ, Nagoya), Yoshihiro Kimura (Kyoto Univ., Kyoto) for fruitful discussions. We also thank Drs. K. Miwa, N. Nakamichi and Todd Michael (Ibis Biosciences, Carlsbad) for experimental materials.

\section{SUPPORTING INFORMATION}

Additional Supporting Information may be found in the online version of this article:

Figure S1. Phylogenetic tree (Neighbor-Joining method) based on atpF-atpH sequences.

Figure S2. Nine strains of five duckweed species in four genera cultured in NF medium.

Figure S3. Bioluminescence of CaMV35S::luc+ in L. gibba p8L under constant light conditions.

Figure S4. Bioluminescence of AtPRR1::luc+ in L. gibba p8L under constant light conditions.

\section{REFERENCES}

Barak S., Tobin E.M., Andronis C., Sugano S., Green R.M. (2000) All in good time: the Arabidopsis circadian clock. Trends in Plant Science 5:517-522. 
Booij-James I.S., Swegle W.M., Edelman M., Mattoo A.K. (2002) Phosphorylation of the D1 photosystem II reaction center protein is controlled by an endogenous circadian rhythm. Plant Physiology 130:2069-2075.

Chen Y.-Y., Wang Y., Shin L.-J., Wu J.-F., Shanmugam V., Tsednee M., Lo J.-C., Chen C.-C., Wu S.-H., Yeh K.-C. (2013) Iron is involved in the maintenance of circadian period length in Arabidopsis. Plant Physiology 161:1409-1420.

Cleland C.F., Briggs W.S. (1969) Gibberellin and CCC effects on flowering and growth in the long-day plant Lemna gibba G3. Plant Physiology 44:503-507.

Czechowski T., Stitt M., Altmann T., Udvardi M.K., Scheible W.-R. (2005) Genome-wide identification and testing of superior reference genes for transcript normalization in Arabidopsis. Plant Physiology 139:5-17.

Dalchau N., Baek S.J., Briggs H.M., Robertson F.C., Dodd A.N., Gardner M.J., Stancombe M.A, Haydon M.J., Stan G.-B., Gonçalves J.M., Webb A.A.R. (2011) The circadian oscillator gene GIGANTEA mediates a long-term response of the Arabidopsis thaliana circadian clock to sucrose. Proceedings of the National Academy of Sciences of the United States of America 108:5104-5109.

Dodd A.N., Dalchau N., Gardner M.J., Baek S.-J., Webb A.A.R. (2014) The circadian clock has transient plasticity of period and is required for timing of nocturnal processes in Arabidopsis. The New Phytologist 201:168-179.

Dodd A.N., Kusakina J., Hall A., Gould P.D., Hanaoka M. (2014) The circadian regulation of photosynthesis. Photosynthesis Research 119:181-190.

Dodd A.N., Salathia N., Hall A., Kévei E., Tóth R., Nagy F., Hibberd J.M., Millar A.J., Webb A.A.R. (2005) Plant circadian clocks increase photosynthesis, growth, survival, and competitive advantage. Science 309:630-633.

Dunlap J.C., Loros J.J., DeCoursey P.J. (2009) Chronobiology: Biological Timekeeping. Sinauer Associates, Inc., Sunderland, MA. 
Fukuda H., Nakamichi N., Hisatsune M., Murase H., Mizuno T. (2007) Synchronization of plant circadian oscillators with a phase delay effect of the vein network. Physical Review Letters 99:098102.

Fukuda H., Ukai K., Oyama T. (2012) Self-arrangement of cellular circadian rhythms through phase-resetting in plant roots. Physical Review E 86:041917.

Gould P.D., Diaz P., Hogben C., Kusakina J., Salem R., Hartwell J., Hall A. (2009) Delayed fluorescence as a universal tool for the measurement of circadian rhythms in higher plants. The Plant Journal 58:893-901.

Graf A., Schlereth A., Stitt M., Smith A.M. (2010) Circadian control of carbohydrate availability for growth in Arabidopsis plants at night. Proceedings of the National Academy of Sciences of the United States of America 107:9458-9463.

Green R.M., Tingay S., Wang Z., Tobin E.M. (2002) Circadian rhythms confer a higher level of fitness to Arabidopsis plants. Plant Physiology 129:576-584.

Hillman W.S. (1961) Experimental control of flowering in Lemna. III. A relationship between medium composition and the opposite photoperiodic responses of L. perpusilla 6746 and $L$. gibba G3. American Journal of Botany 48:413-419.

Hillman W.S. (1970) Carbon dioxide output as an index of circadian timing in Lemna photoperiodism. Plant Physiology 45:273-279.

Ishiura M., Kutsuna S., Aoki S., Iwasaki H., Andersson C.R., Tanabe A., Golden S.S., Johnson C.H., Kondo T. (1998) Expression of a gene cluster kaiABC as a circadian feedback process in cyanobacteria. Science 281:1519-1523.

Kojima S., Shingle D.L., Green C.B. (2011) Post-transcriptional control of circadian rhythms. Journal of Cell Science 124:311-320.

Kondo T., Tsudzuki T. (1978) Rhythm in potassium uptake by a duckweed, Lemna gibba G3. Plant \& Cell Physiology 19:1465-1473. 
Kusakina J., Gould P.D., Hall A. (2014) A fast circadian clock at high temperatures is a conserved feature across Arabidopsis accessions and likely to be important for vegetative yield. Plant, Cell \& Environment 37:327-340.

Landolt E. (1986) Biosystematic Investigations on the Family of Duckweeds: The Family of Lemnaceae - A Monograph Study. Veröffentlichungen des Geobotanischen Instituts ETH, Stiftung Rübel, Zürich, Switzerland.

Leeuwen W., Hagendoorn M.J.M., Ruttink T., Poecke R., Plas L.H.W., Krol A.R. (2012) The use of the luciferase reporter system for in planta gene expression studies. Plant Molecular Biology Reporter 18:143-144.

Les D.H., Landolt E., Crawford D.J. (1997) Systematics of the Lemnaceae (duckweeds): Inferences from micromolecular and morphological data. Plant Systematics and Evolution 204:161-177.

McClung C.R. (2013) Beyond Arabidopsis: the circadian clock in non-model plant species. Seminars in Cell \& Developmental Biology 24:430-436.

Michael T.P., Salomé P.A., Yu H.J., Spencer T.R., Sharp E.L., McPeek M. a, Alonso J.M., Ecker J.R., McClung C.R. (2003) Enhanced fitness conferred by naturally occurring variation in the circadian clock. Science 302:1049-1053.

Millar A.J., Short S.R., Chua N.H., Kay S.A. (1992) A novel circadian phenotype based on firefly luciferase expression in transgenic plants. The Plant Cell 4:1075-1087.

Miwa K., Serikawa M., Suzuki S., Kondo T., Oyama T. (2006) Conserved expression profiles of circadian clock-related genes in two Lemna species showing long-day and short-day photoperiodic flowering responses. Plant \& Cell Physiology 47:601-612.

Miyata H., Yamamoto Y. (1969) Rhythms in respiratory metabolism of Lemna gibba G3 under continuous illumination. Plant \& Cell Physiology 10:875-889.

Muranaka T., Kubota S., Oyama T. (2013) A single-cell bioluminescence imaging system for monitoring cellular gene expression in a plant body. Plant \& Cell Physiology 54:2085-2093. 
Nakamichi N. (2011) Molecular mechanisms underlying the Arabidopsis circadian clock. Plant \& Cell Physiology 52:1709-1718.

Nakamichi N., Ito S., Oyama T., Yamashino T., Kondo T., Mizuno T. (2004) Characterization of plant circadian rhythms by employing Arabidopsis cultured cells with bioluminescence reporters. Plant \& Cell Physiology 45:57-67.

Ohta S., Mita S., Hattori T., Nakamura K. (1990) Construction and expression in Tobacco of a $\beta$-Glucuronidase (GUS) reporter gene containing an intron within the coding sequence. Plant \& Cell Physiology 31:805-813.

Pal S.K., Liput M., Piques M., Ishihara H., Obata T., Martins M.C.M., Sulpice R., van Dongen J.T., Fernie A.R., Yadav U.P., Lunn J.E., Usadel B., Stitt M. (2013) Diurnal changes of polysome loading track sucrose content in the rosette of wild-type arabidopsis and the starchless pgm mutant. Plant Physiology 162:1246-1265.

Park K.H., Hahn K.W., Kang B.G. (1990) Lack of correlation between senescence and stomatal aperture in Lemna gibba G3. Plant \& Cell Physiology 31:731-733.

Plautz J.D., Straume M., Stanewsky R., Jamison C.F., Brandes C., Dowse H.B., Hall J.C., Kay S.A. (1997) Quantitative analysis of Drosophila period gene transcription in living animals. Journal of Biological Rhythms 12:204-217.

Pokhilko A., Mas P., Millar A.J. (2013) Modelling the widespread effects of TOC1 signalling on the plant circadian clock and its outputs. BMC Systems Biology 7:23-34.

Serikawa M., Miwa K., Kondo T., Oyama T. (2008) Functional conservation of clock-related genes in flowering plants: overexpression and RNA interference analyses of the circadian rhythm in the monocotyledon Lemna gibba. Plant Physiology 146:1952-1963.

Song Y.H., Ito S., Imaizumi T. (2010) Similarities in the circadian clock and photoperiodism in plants. Current Opinion in Plant Biology 13:594-603. 
Wang W., Wu Y., Yan Y., Ermakova M., Kerstetter R., Messing J. (2010) DNA barcoding of the Lemnaceae, a family of aquatic monocots. BMC Plant Biology 10:205-215.

Welsh D.K., Imaizumi T., Kay S.A. (2005) Real-time reporting of circadian-regulated gene expression by luciferase imaging in plants and mammalian cells. Methods in Enzymology 393:269-288.

Yakir E., Hassidim M., Melamed-Book N., Hilman D., Kron I., Green R.M. (2011) Cell autonomous and cell-type specific circadian rhythms in Arabidopsis. The Plant Journal 68:520-531.

Yukawa I., Takimoto A. (1976) Flowering response of Lemna paucicostata in Japan. The Botanical Magazine Tokyo 89:241-250. 


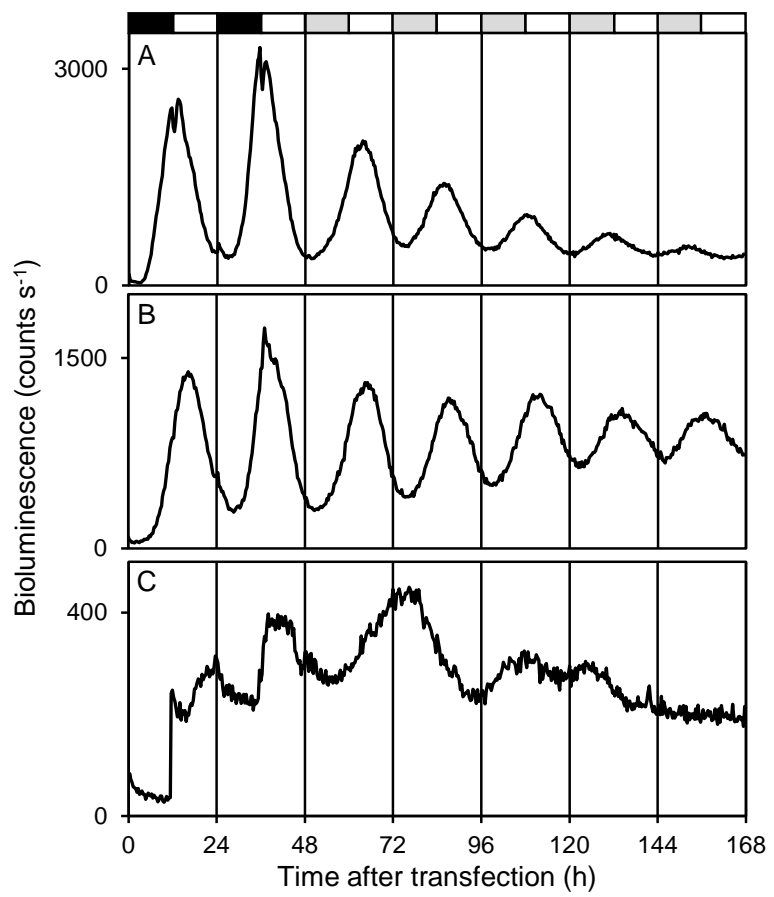

Fig. 1 Temporal bioluminescence changes of transfected duckweed plants. The circadian reporter AtCCA1::luc+ was introduced into L. gibba G3 cultured in E medium (A), L. gibba G3 cultured in $0.5 \times \mathrm{H}$ medium (B), S. polyrhiza 7003 cultured in $0.5 \times \mathrm{H}$ medium (C). Plants cultured under constant light conditions were subjected to gene transfection by particle bombardment and then entrained to two $12 \mathrm{~h}$ dark / $12 \mathrm{~h}$ light cycles and released into constant light conditions. Black, open, and gray bars indicate dark, light, and subjective night, respectively. Data are representative of $\mathrm{n}=4$ replicate dishes. 

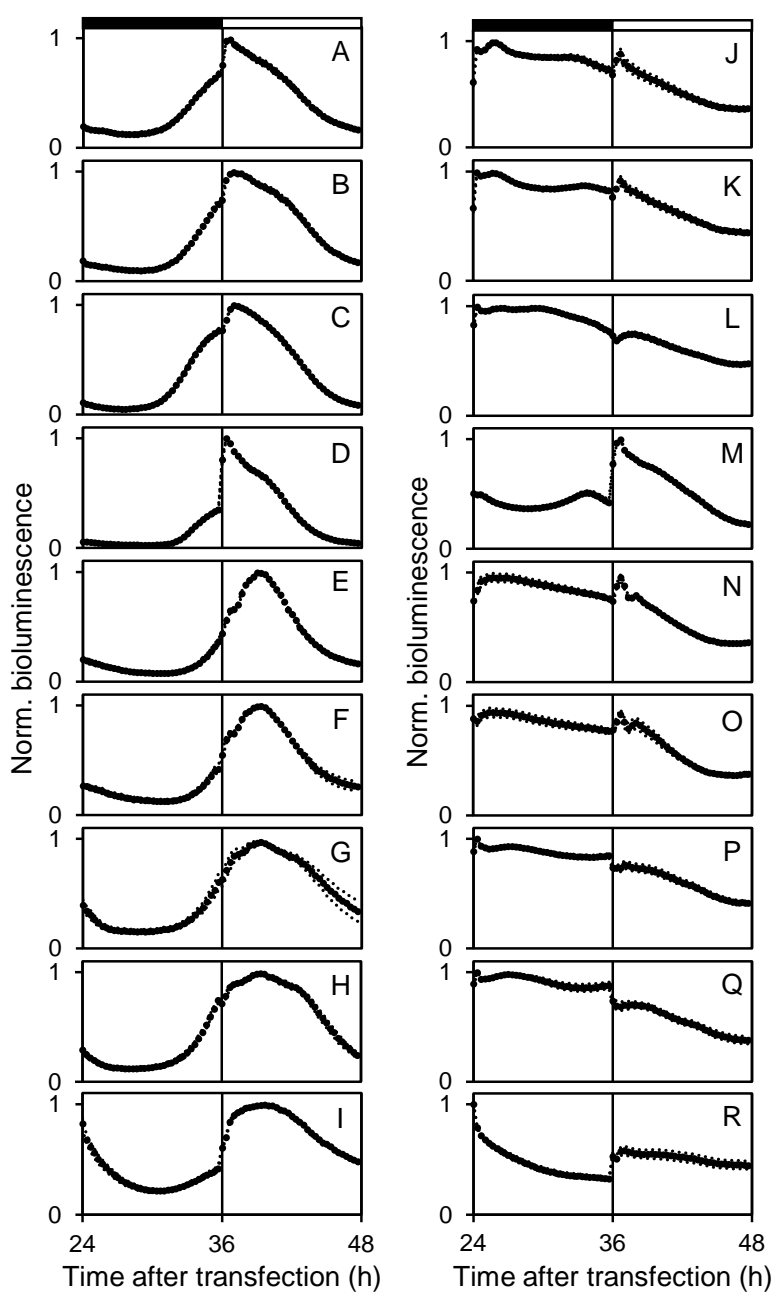

Fig. 2 Bioluminescence of AtCCA1::luc+ and ZmUBQ1::luc+ under the $12 \mathrm{~h}$ dark / $12 \mathrm{~h}$ light cycle. AtCCA1::luc+ was introduced into L. gibba G3 (A) and p8L (B), L. aequinoctialis 6746 (C) and Nd (D), L. punctata NS (E) and IS (F), S. polyrhiza 7003 (G) and biwa1 (H), W. columbiana 7972 (I). ZmUBQ1::luc+ was introduced into L.gibba G3 (J) and p8L (K), L. aequinoctialis 6746 (L) and Nd (M), L. punctata NS (N) and IS (O), S. polyrhiza 7003 (P) and biwa1 (Q), W. columbiana 7972 (R). Plants cultured in NF medium under constant light conditions were subjected to gene transfection and then transferred to $12 \mathrm{~h}$ dark / $12 \mathrm{~h}$ light conditions. Bioluminescence intensities 24-48 h after gene transfection were plotted. Bioluminescence intensities were normalized by dividing by their 24-48 h maximum. Mean values (black circle) \pm SEM (dotted line) of $n=8$ replicate dishes were plotted. Black and open bars indicate dark and light, respectively. 

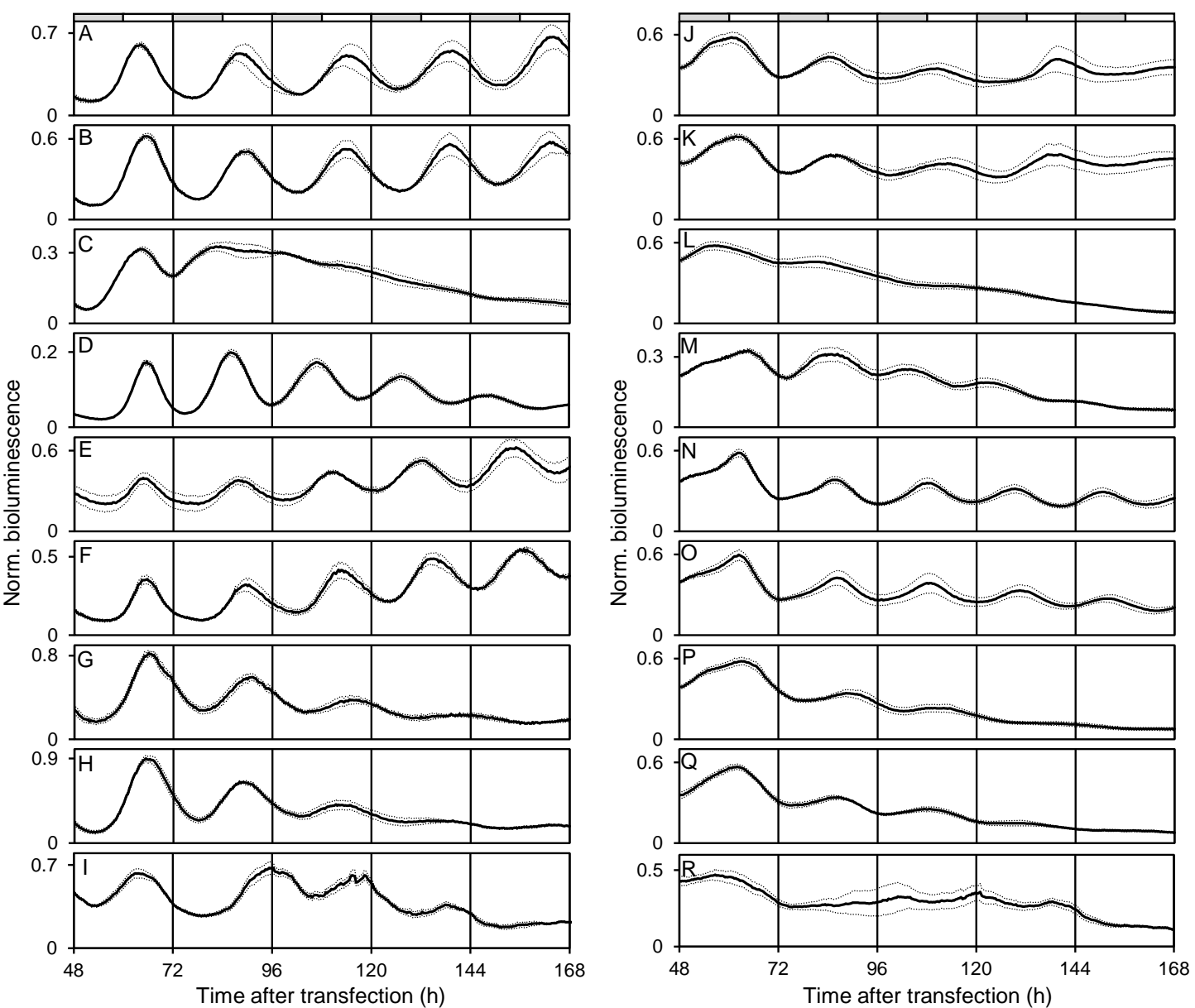

Fig. 3 Bioluminescence of AtCCA1::luc+ and ZmUBQ1::luc+ under constant light conditions. AtCCA1::luc+ was introduced into L. gibba G3 (A) and p8L (B), L. aequinoctialis 6746 (C) and Nd (D), L. punctata NS (E) and IS (F), S. polyrhiza 7003 (G) and biwa1 (H), W. columbiana 7972 (I). ZmUBQ1::luc+ was introduced into L. gibba G3 (J) and p8L (K), L. aequinoctialis 6746 (L) and Nd (M), L. punctata NS (N) and IS (O), S. polyrhiza 7003 (P) and biwa1 (Q), W. columbiana 7972 (R). Plants cultured in NF medium under constant light conditions were subjected to gene transfection and then entrained to two $12 \mathrm{~h}$ dark / $12 \mathrm{~h}$ light cycles and released into constant light conditions. Bioluminescence intensities 48-168 h after gene transfection were plotted. Bioluminescence intensities were normalized by dividing by their $24-48 \mathrm{~h}$ maximum. Mean values (solid line) \pm SEM (dotted line) of $n=4$ replicate dishes were plotted. Open and gray bars indicate light and subjective night, respectively. 

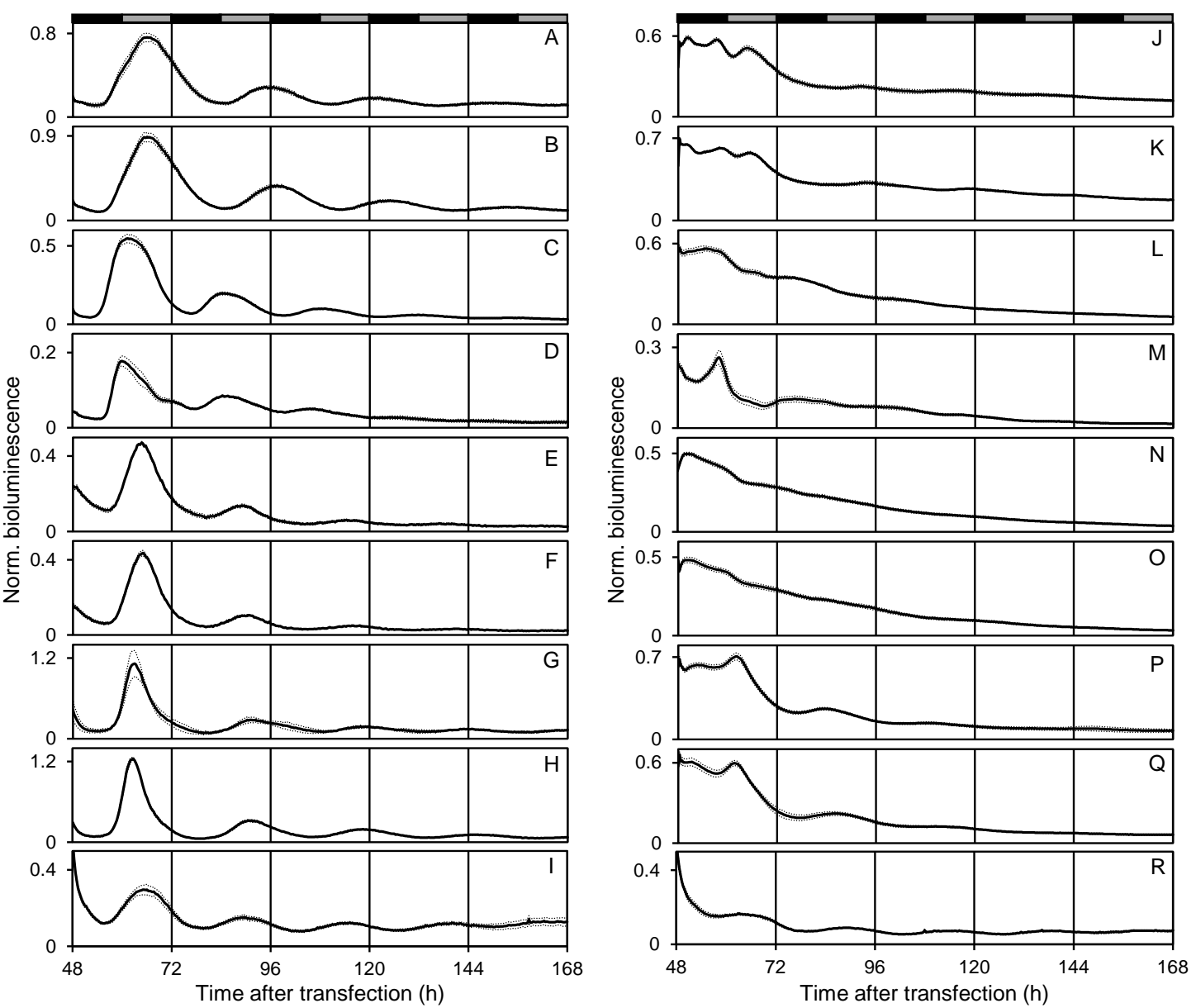

Fig. 4 Bioluminescence of AtCCA1::luc+ and ZmUBQ1::luc+ under constant dark conditions. AtCCA1::luc+ was introduced into L. gibba G3 (A) and p8L (B), L. aequinoctialis 6746 (C) and Nd (D), L. punctata NS (E) and IS (F), S. polyrhiza 7003 (G) and biwa1 (H), W. columbiana 7972 (I). ZmUBQ1::luc+ was introduced into L. gibba G3 (J) and p8L (K), L. aequinoctialis 6746 (L) and Nd (M), L. punctata NS (N) and IS (O), S. polyrhiza 7003 (P) and biwa1 (Q), W. columbiana 7972 (R). Plants cultured in NF medium under constant light conditions were subjected to gene transfection and then entrained to two $12 \mathrm{~h}$ dark / $12 \mathrm{~h}$ light cycles and released into constant dark conditions. Bioluminescence intensities of 48-168 h after gene transfection were plotted. Bioluminescence intensities were normalized by dividing by their 24-48 h maximum. Mean values (solid line) \pm SEM (dotted line) of $n=4$ replicate dishes were plotted. Black and gray bars indicate dark and subjective day, respectively. 


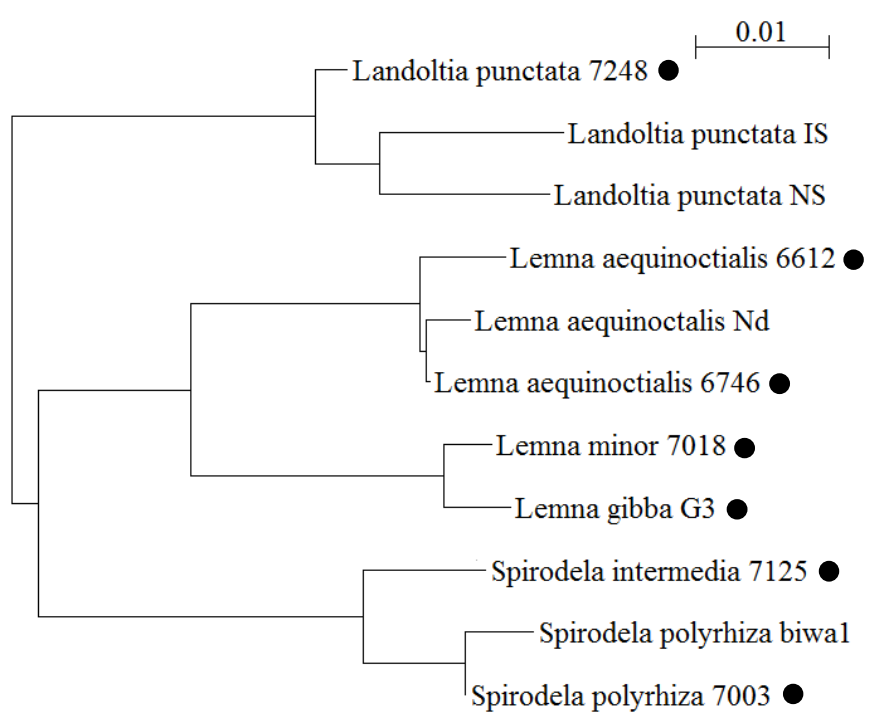

Supplementary Fig. S1 Phylogenetic tree (Neighbor-Joining method) based on atpF-atpH sequences. Black dots indicate reference strains reported in Wang et al. (2010). 

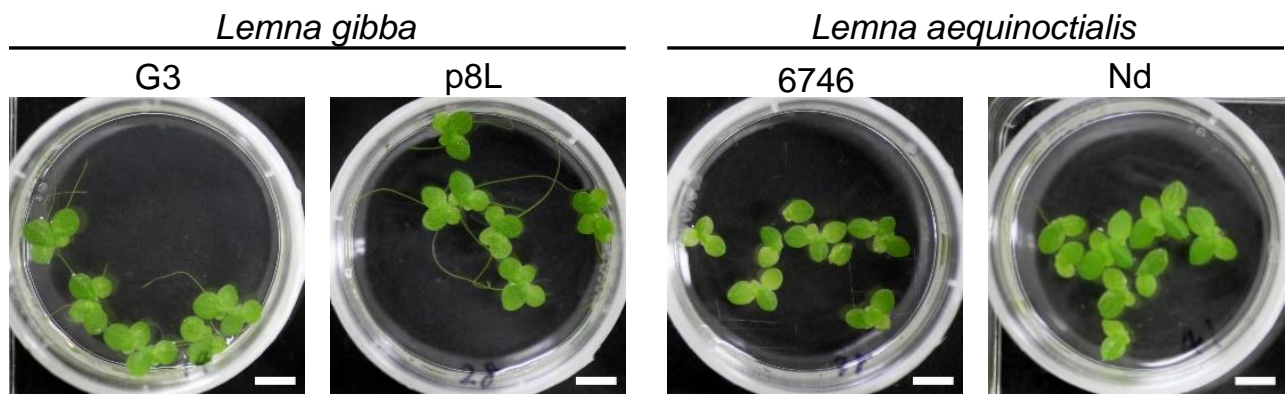

Landoltia punctata
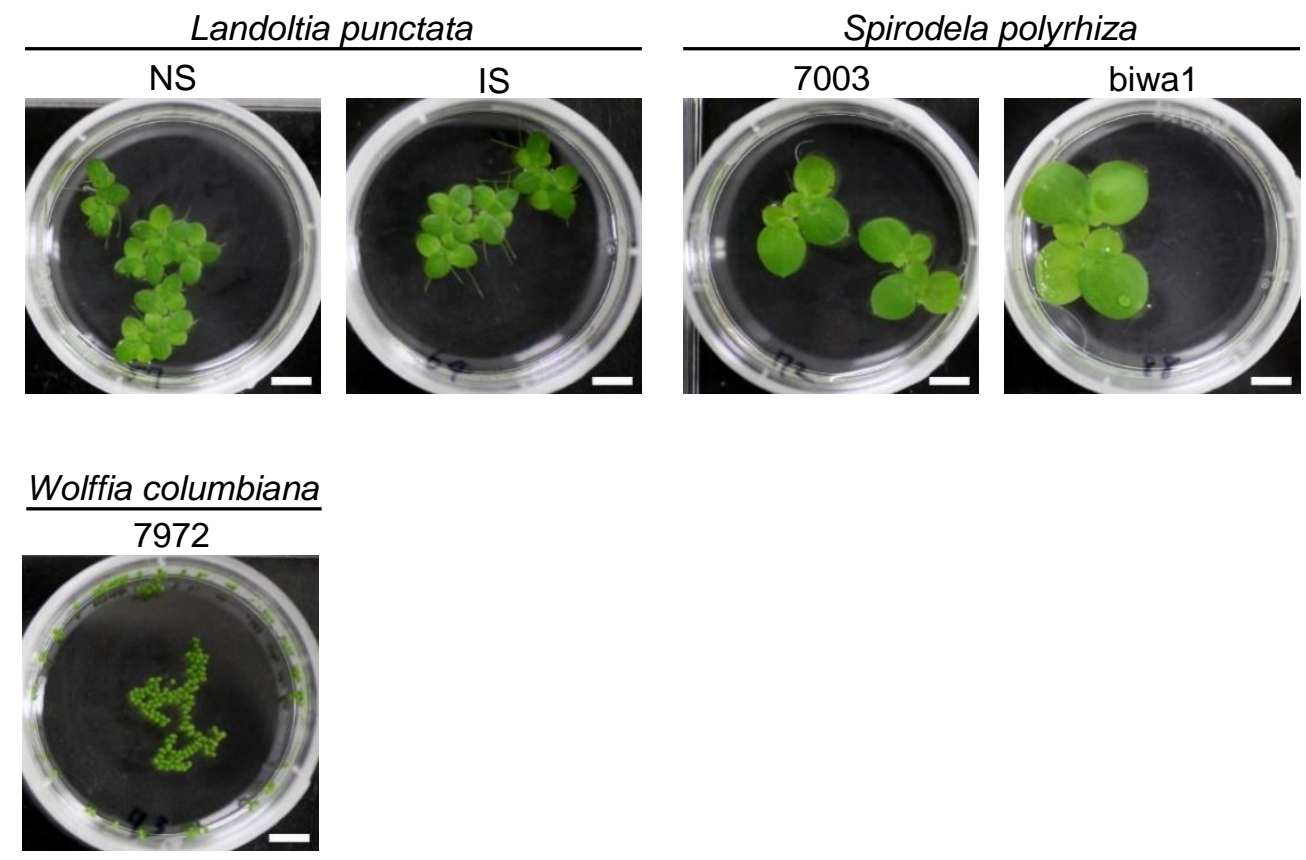

Supplementary Fig. S2 Nine strains representing five duckweed species across four genera cultured in NF medium. Pictures were taken just after gene transfection. Plants were placed in 35-mm dishes for bioluminescence monitoring. Scale bars indicate $5 \mathrm{~mm}$. 


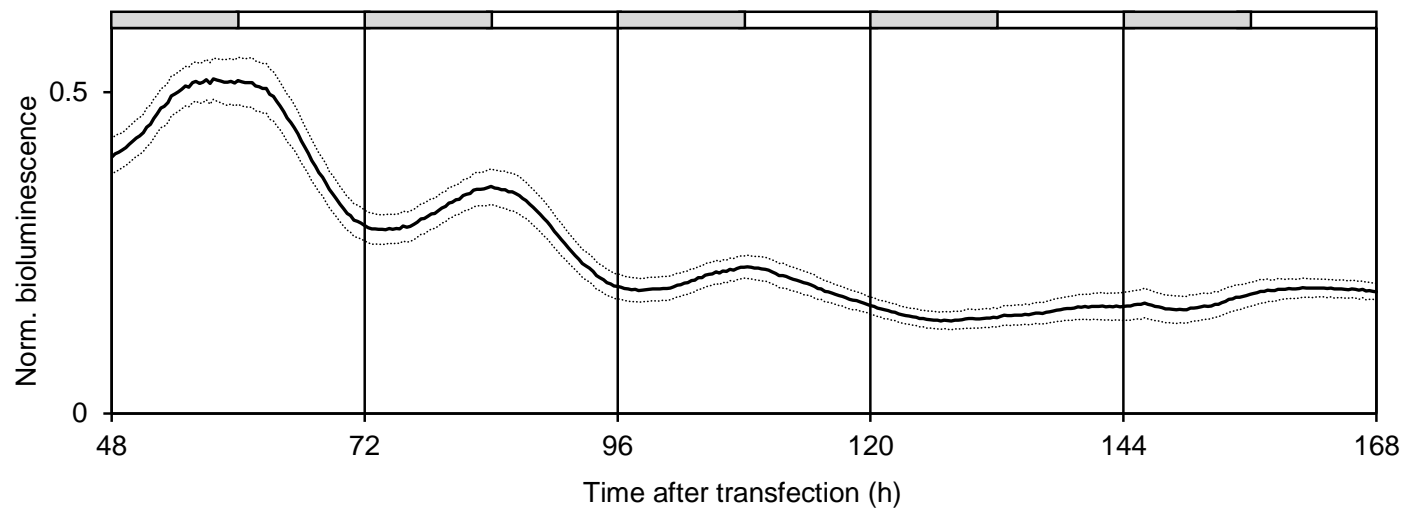

Supplementary Fig. S3 Bioluminescence of CaMV35S::luc+ in L. gibba p8L under constant light conditions. Plants cultured in NF medium under constant light conditions were subjected to gene transfection and then entrained to two $12 \mathrm{~h}$ dark / $12 \mathrm{~h}$ light cycles and released into constant light conditions. Bioluminescence intensities of $48-168 \mathrm{~h}$ after gene transfection were plotted. Bioluminescence intensities were normalized by dividing by their $24-48 \mathrm{~h}$ maximum. Mean values (solid line) \pm SEM (dotted line) of $n=4$ replicate dishes were plotted. Open, gray bars indicate light, and subjective night, respectively. 


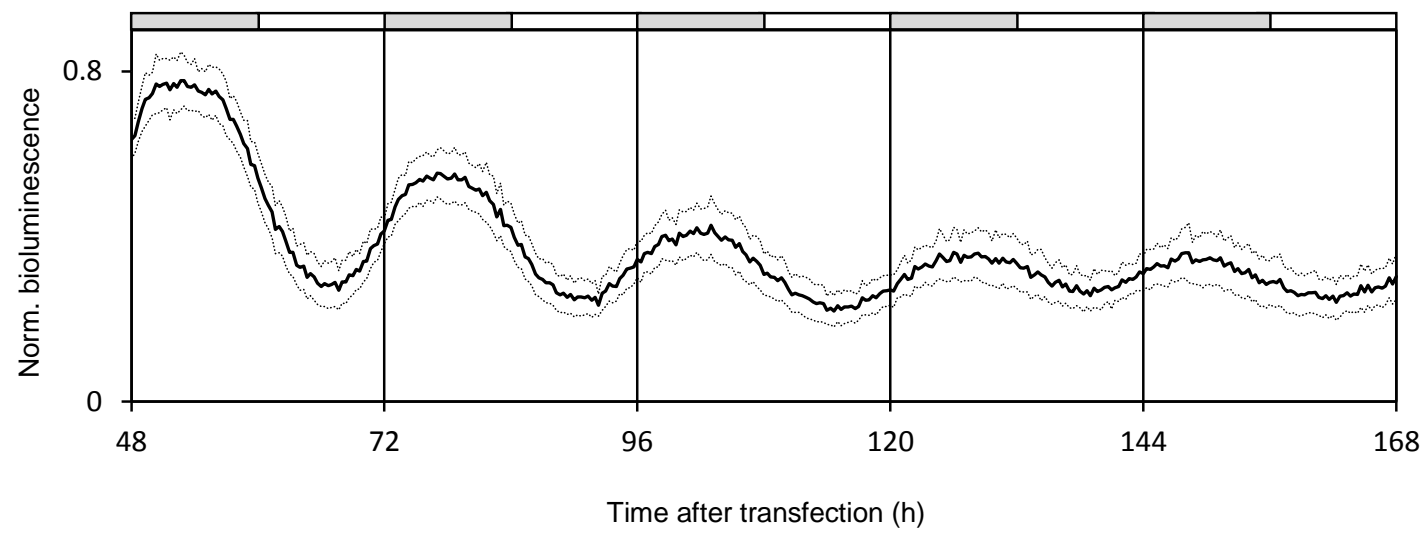

Supplementary Fig. S4 Bioluminescence of AtPRR1::luc+ in L. gibba p8L under constant light conditions. Plants cultured in NF medium under constant light conditions were subjected to gene transfection and then entrained to two $12 \mathrm{~h}$ dark / $12 \mathrm{~h}$ light cycles and released into constant light conditions. Bioluminescence intensities of $48-168 \mathrm{~h}$ after gene transfection were plotted. Bioluminescence intensities were normalized by dividing by their $24-48 \mathrm{~h}$ maximum. Mean values (solid line) \pm SEM (dotted line) of $n=4$ replicate dishes were plotted. Open, gray bars indicate light, and subjective night, respectively. 